\title{
Acute psychiatric ward rules: a review of the literature
}

\author{
J. ALEXANDER ${ }^{1}$ RMN BA (Hons) MA \& L. BOWERS ${ }^{2}$ RMN PhD \\ ${ }^{1}$ Lecturer in Psychology \& Communication Skills, and ${ }^{2}$ Professor of Psychiatric Nursing, City University, St \\ Bartholomew School of Nursing \& Midwifery, Department of Mental Health and Learning Disabilities, London, UK
}

Correspondence:

J. Alexander

City University

St Bartholomew School of Nursing

\& Midwifery

Department of Applied

Psychosocial Sciences

20 Bartholomew Close

London

EC1A $7 Q N$

UK

E-mail:E.J.Alexander@city.ac.uk

\section{ALEXANDER J. \& BOWERS L. (2004) Journal of Psychiatric and Mental Health Nursing} 11, 623-631

\section{Psychiatric ward rules: a literature review}

This literature review forms a background element of a comparative study of two acute psychiatric wards in the East End of London. The research focused on ward rules as a means of investigating the relationship between the flexibility/inflexibility of ward nursing regimes and patient outcomes. Previous studies identified a relationship between ward rules and patient aggression. Other studies identified a link between absconding by inpatients and nurses' attitudes towards rule enforcement. However, an in-depth exploration of psychiatric ward rules from the perspective of nurses and patients has not been undertaken previously.

Keywords: acute inpatient care, acute psychiatric ward nursing regimes, flexibility, inflexibility, patient outcomes, ward rules

Accepted for publication: 26 April 2004

\section{Introduction}

Psychiatric nurses employ rules to set limits on patients' behaviour, for example on smoking, sexual appropriateness and time of return to the ward. Rules are a tangible means of assessing the flexibility or inflexibility of the ward social system because the way in which they are applied and the relative importance attached to them, indicates the responsiveness of nursing regimes towards patients' needs. Rigidity may reflect the intractability of ward systems and the inability to provide individualized nursing care in the form of one-to-one contact with patients (Pilgrim \& Rogers 1994).

Since the inception of the hospital closure programme and the advent of care in the community, huge changes have occurred within psychiatry. Firstly, the function of acute admission wards has altered radically. Bed shortages and emphasis on acuity have put pressure on nurses to contain and intensively treat psychotic patients (Taylor \& Taylor 1989, Thomas 1996). Consequently, ward rules may be implemented rigidly because patients are expected to comply rapidly with the treatment regimen so that they can be discharged.
The Department of Health (DOH 2001) recommended that a code of conduct for inpatients should incorporate behavioural expectations. The code should also contain guidance on ward rules, which should be negotiated with service users, and they should be given written information upon reception to the wards. The $\mathrm{DOH}$ also identified a need for regular forums that encourage service-user involvement in deciding how the ward is organized, and recommended that they address appropriate rules for patient conduct.

\section{Search methods}

A search of CINAHL and PSYCHLIT electronic databases yielded 660 nursing articles related to ward rules and structure, 198 of which were selected and reviewed. The search terms used were: rule, ward, regime, atmosphere, routine, management, structure, boundaries, limit setting, disobedient, discipline, authority, conform, compliance, noncompliance, non-cooperative, resist, norm, regulate, order, control, restrict, conduct. Not all were relevant, but those that were appropriate were analyzed in chronological order of publication. This mode of analysis was chosen because it 
tracked institutional changes within psychiatry, which might be reflected at ward level in nurses' attitudes towards control.

\section{The literature}

\section{7}

A comparative study by Jungman \& Bucher (1967) evaluated the influence of ward milieu on patient behaviour. They concluded that differing ward management systems in terms of rule clarity, structure and defined staff roles accounted for variations in the frequency of rule breaking between the two wards in the study. However, one of the wards contained a group of long-stay chronic patients, and this might have confounded the results. Arguably, the particular clinical management problems with this patient group rather than the ward social system alone could account for differences in outcomes.

\section{1}

In a descriptive study, Jurgensen (1971) advocated consistent limit setting in the management of hospitalized adolescent patients, and perceived that this approach should not be confined to the management of disruptive or antisocial behaviour, rather it should form an intrinsic part of the patient's care plan, and not be confined to the enforcement of hospital rules.

\section{6}

Similarly, Bursten \& Geach (1976) analyzed whether the step system was justified on therapeutic grounds. This system curtails patients' autonomy in order to control their behaviour, and aims to resocialize them by using a graded series of privileges or activities. Rule breaking and the step system are interlinked; for example, patients who leave the ward without permission may be grounded, so that they learn to ask permission in the future. Only one of the study wards operated a formal step system, but no differences in patient outcomes were found, and the authors questioned whether the use of the formal step system was therapeutically justified. The study was limited because it used a retrospective method to evaluate therapeutic outcomes, but the results contrasted with Jungman \& Bucher's (1967) findings, in which high structure improved patient compliance.

\section{8}

Alden (1978) recorded nurse-patient interactions, and used the Ward Atmosphere Scale (WAS) to measure patients' perceptions of the psychosocial environments of eight psychiatric wards. The WAS (Moos 1979) contains eight dimensions, one of which has a subscale on control, which measures how strictly nurses enforce rules and schedules. Certain patients tended to be withdrawn, and they perceived the staff as very rigid, but they expressed less hostility than those patients within wards where the expression of anger was less strictly controlled. These patients were more communicative, but they also tended to be more hostile and independent. This study elaborated on previous articles by focusing on specific patient outcomes produced by different ward nursing regimes. Jungman \& Bucher (1967) and Bursten \& Geach (1976) provided a generalized behaviourist overview. Alden (1978) highlighted the negative psychosocial effects of rigid regimes, and demonstrated that the achievement of patient compliance may impede recovery.

\section{9}

Levinson \& Crabtree (1979) found that adherence to the ward structure was helpful in the management of crises in a therapeutic community setting for hospitalized adolescents. However, the findings were based on the authors' experiences within one unit with no systematic methods of data collection. A similar study by Watkins (1979) discussed role ambivalence amongst residential and medical staff about the use of authority. He argued for rule clarity, and interdisciplinary decision making in order to integrate the use of authority in treatment.

\section{0}

Bursten et al. (1980) analyzed the therapeutic value of restrictive and coercive ward policies in a comparative study of patient outcomes on three wards. Ward A operated a formal step system, Ward B1 was highly restrictivecoercive, and compliance with treatment was mostly optional in Ward B3. Nurses, patients and family members rated outcomes during admission and at discharge. Patients and their significant others rated outcomes at 6month follow-up. Ward A patients showed better adjustment than those in Ward B1 or Ward B3 patients, and Ward B1 patients' outcomes surpassed those of the Ward B3 sample. However, a lack of inter-rater reliability may have skewed the results and the ratings may have been influenced by the patients' close relationships with their significant others. The self-rating patients may have lacked objectivity. Further, they may have feared the consequences of reporting their actual levels of functioning, or an overly compliant group may have responded. Hypothetically, the rest of the patient sample might report 
accurately because their inpatient experiences were less coercive and restrictive. The study reflects and expands on Jungman \& Bucher's (1967) findings. However, they conflict with Bursten \& Geach's (1976) results, which failed to identify different patient outcomes, but this study was restricted to inpatients. Bursten et al.'s (1980) findings also contrast with Alden's (1978) results in which patient outcomes were adversely effected by rigid ward nursing regimes, but Alden's sample was larger and restricted to inpatients. Alden's study was important because it began to question the role of staff attitudes, and the influence of nurse-patient communication.

\section{1}

Flaherty et al. (1981) used WAS as a means of testing whether black and white patients within the same ward perceived the atmosphere differently or not. The white patients' duration of stay was longer, and more black patients left the ward against medical advice. White patients also enjoyed more autonomy, and the authors hypothesized that treatment variance might have influenced differing perceptions of the ward atmosphere between the two groups. They acknowledged that cultural factors might have influenced the black patients' negative perceptions of the ward atmosphere in that they might have different expectations of the ward environment, which might have caused them to react negatively during admission. Further, they hypothesized that staff may have responded to this behaviour with increased defensiveness. However, although diagnoses were matched, the samples were undifferentiated by chronicity, and this could explain some of the black patients' responses towards the environment.

\section{3}

Johansen (1983) discussed the behaviour of personalitydisordered patients, and observed that their low tolerance for frustration caused them to disregard ward rules frequently. He described a situation in which the ward became less flexible following an arson incident, and recommended constant flexibility to facilitate individualized care. He warned that chronically ill patients might be neglected when nurses impose limits on patients with personality disorder who use the rules to gain more attention. He also recommended greater structure, but this may be difficult to combine with rule flexibility in the management of personality disorder. Johansen's views contrasted with Levinson \& Crabtree's (1979) recommendations in that they advocated constant adherence to the rules in order to avoid arbitrary decision making during ward crises.

\section{8}

Lanza (1988) studied the relationship between ward rules and patient assaults, and found that many incidents (32\%) occurred within a limit setting context, or during the daily ward routine. Nurses' recall for the antecedents of the incidents was patchy, but they reported that many attacks occurred in silence, without warning and with intent to do harm. Lanza leant towards a psychopathologic explanation of patient aggression, but the retrospective design, and the grouping of diverse behaviours meant that there was no way of identifying whether aspects of the interaction triggered incidents or not. The findings contrasted with Alden's (1978) study in that, despite the emphasis on high structure, the staff did not intimidate the patients. This also reflected the reactions of patients, who were cared for in the less restrictive wards in Alden's study, but Alden recorded nurse-patient interactions, and Lanza studied violent incidents rather than patients' perceptions of the ward atmosphere. The patient samples also differed in that Lanza included psycho-geriatric groups, and their misperceptions of the environment might have triggered aggression. Despite this, it does appear that the nurses in Lanza's study might not have been overcontrolling, and if so, psychopathology could have been a factor in aggression.

\section{9}

Ward rules were central to a theoretical framework for patient violence devised by Morrison (1989). She hypothesized that patient aggression was associated with lack of rule clarity and inconsistency. This hypothesis was supported by the results in that Morrison found that a rise in the inconsistent application of the rules was associated with an increase in non-compliance. She used the results to generate five predictors of violence, which included the discrepant interpretation of the therapeutic rules, the inconsistent enforcement of the social rules, a diagnosis of schizophrenia or substance abuse, and the patients' inability to adhere to the social rules.

Psychodynamic theory offers an alternative perspective on the role of ward rules in patient care. Kologjera et al. (1989) used this to describe the therapeutic use of seclusion in the management of disruptive adolescent behaviour. They held that insecure parental boundaries did not allow children to experiment safely with new behaviours in socially acceptable ways. The non-compliance by adult psychiatric patients may also represent an attempt to provoke parental figures to provide clearly defined boundaries, and a holding environment. Kologjera et al. argued that rule consistency could help patients test boundaries within a secure and supportive environment. They perceived that 
abused children compulsively repeat early experiences of abuse because they learned to provoke punitive parental attention as a means of maintaining closeness at an early stage of development. Nurses could be drawn into repetitions of similar punitive responses towards non-compliant patients, but they could provide a corrective emotional experience through appropriate responses wherein the patient learns that support and discipline need not be punitive. This study elaborated on recommendations by Jurgensen (1971), Levinson \& Crabtree (1979), and Watkins (1979), and reflected similar ward management principles. These studies contrast with Johansen's (1983) theory, which advocated rule flexibility in the management of ward crises.

\section{0}

Lutzen (1990) found that the dominant psychiatric ideology conflicted with nurses' moral values. Four of the categories that emerged from the data were concerned with written or unwritten rules. When they complied with the authority of the hospital, nurses followed the written rules, but they subverted this when they followed the unwritten rules, and came to an implicit agreement that in practice, all the written rules could not be adhered to. Nurses felt that controlling activities conflicted with their basic values, and they felt that they could not provide authentic nursing care. They managed role ambivalence by maintaining professionalism, and interpreted this as distancing themselves from patients. Group alliance was another way in which they coped with ideological conflict.

\section{1}

Roper \& Anderson (1991) found that patient violence was associated with nurses' attitudes towards control. The results showed that staff used the ward structure and the denial of patients' requests on order to maintain control. The authors made negative interpretations of limit setting, and seemed to divorce this from the care context. The authors' perspective contrasted with that of Kologjera et al.'s (1989) views and those of Levinson \& Crabtree's (1979) who advocated firm boundaries in the management of patients.

In a descriptive study, De Laune (1991) wrote about the management of manipulative patients. She discussed the way that nurses tend to label this type of behaviour, and avoid manipulative patients. This increases patients' anxiety, and reinforces the behaviour. She advocated limit setting as the most effective way of managing various types of manipulation with the aim of:

- establishing boundaries;
- preventing escalation; and

- counteracting resistance.

This study strengthened the recommendations of Jurgensen (1971), Levinson \& Crabtree (1979), Watkins (1979), and Kologjera et al. (1989), and reflected similar ward management principles, which conflict with Johansen's (1983) theory about rule flexibility in the management of ward crises.

\section{3}

Patients may not perceive ward rules as punitive or controlling, and may view boundary setting as an expression of nurses' support or concern. Caplan (1993) used WAS to measure the influence of the ward environment on patients' perceptions of control. The results highlighted differences between the nurses' and patients' perceptions of rule clarity. Many patients were unclear about rules and behavioural expectations. They also perceived higher levels of control than the staff, but both groups identified good levels of support within the environment. Caplan suggested that patients viewed control positively because it was imposed within a caring context. This hypothesis reflected Kologjera et al.'s (1989) theory about the efficacy of a holding environment for patient management.

\section{4}

Lanza et al. (1994) investigated the relationship between patient autonomy, ward rules and assault. The authors argued that ward rules reflect basic issues about patient autonomy and the extent of staff control. The ward with the highest number of assaults in the study scored lowest on autonomy. Conversely, the ward with the least number of assaults scored highest on autonomy, practical orientation and personal problem orientation. This ward also had the lowest score on staff control, and the ward with the highest rates of assault also had the greatest reported levels of control. The authors advocated the incorporation of therapeutic community ideals within psychiatric wards so that rule consistency is combined with high engagement. Lanza's et al.'s arguments for consistency corresponded with those of Morrison (1989), but they found that autonomy and low staff control produced fewer assaults. These results contrasted with Alden's (1978) findings in which similar factors increased patient hostility.

In 1994, Morrison (1994) discussed the conclusions of her 1989 study, and found that they conflicted with an earlier pilot study, in which nurses associated inconsistency with violence. The findings failed to predict a significant degree of violence, which led her to conclude that consistency might not be vitally important, and this brought into 
question the value of violence prevention methods based on consistency. She perceived that the model had poor predicative power, and this may have been caused by design problems. The nursing staff sample was split almost equally between qualified nurses and aides. Both groups might have approached their patients differently, and they might have made different interpretations of patient behaviour. Diverse behaviours were grouped together, and it was difficult to identify which specific aspects of nurse-patient interaction influenced patient outcomes. The use of quantitative methods to assess qualitative interactions seems problematic. This study elaborated on previous work that advocated persistent and clear limit setting for psychiatric patients, but also casts doubt on their recommendations (Jungman \& Bucher 1967, Levinson \& Crabtree 1979, Bursten et al. 1980).

\section{5}

The importance of rule clarity emerged in Bensley et al.'s (1995) study, which compared the views of hospital staff and patients about ward regimes. The results showed that patients and staff shared many concerns, but patients alone identified a lack of rule clarity as a primary factor in influencing assaults. The study recommendations reflected Morrison's (1989) suggestions about the importance of rule clarity and consistency. Ward rules may be used to exert power over patients.

Hewison (1995) argued that nurses controlled interactions, gave orders routinely and used language to exert power over patients. However, the study was conducted on one care of the elderly ward over a 3-month period, and the author generalized the findings to embrace all nursepatient interactions within psychiatry.

Richardson (1995) argued that rules for patient conduct must be properly devised, and established by statute in consultation with patients. Rule justification and the exercise of authority must be directly associated with institutional requirements to maintain safety and control. A formal system of sanctions may not be appropriate for acute mental health units, but a modified version of the process might provide a consistent, and less arbitrary response to rule breaking by patients.

A project by Morales (1995) aimed to decrease the use of restraints and seclusion within an acute unit through prevention and early intervention. The use of restrictive measures decreased by $50 \%$ through the use of verbal deescalation, medication, limit setting, quiet time and listening to soft music. However, it is unclear whether the study that took place on one ward for chronic psychiatric inpatients could be generalized to embrace acute wards with high levels of patient acuity and turnover.

\section{6}

In a study of the use of seclusion, Muir-Cochrane \& Harrison (1996) used grounded theory to gather data, and a core category 'controlling' emerged in the practice of seclusion. They found that staff set arbitrary limits based on the patients' individual responses to individual nurses. The authors acknowledged that the findings were not generalizable, but they argued that they provided a rich insight into the social processes involved in the use of seclusion. However, it was unclear which behaviours were restricted because diverse behaviours were grouped together, which meant that information on the antecedents and consequences of individual incidents was lacking. The study echoed the findings of Roper \& Anderson (1991) in which staff made constant references about the need to control patients.

The number of ward rules may reflect the ward social climate. Aubrey et al. (1996) used WAS to measure the impact of environmental changes on staff and patients in an acute psychiatric unit, which had relocated from a mental institution to a general hospital site. Specific changes were implemented, which included rule clarity, fewer searches, door locking and the flexible use of the step system. It was difficult to ascertain how these changes were achieved, but patients perceived improvements in four out of five social climate areas of the WAS. However, nurses perceived that they had less control over the ward. This study augmented previous research that advocated rule clarity (Morrison 1989, Bensley et al. 1995).

The way that nurses interact with patients during the course of their duties is associated with violence, and this includes rule enforcement. Whittington \& Wykes (1996) analyzed how often violence was preceded by a nursing approach or demand that patients perceived as unpleasant. They identified three main precipitants of violence, which included frustration, perceived attack and activity demand. They found that $86 \%$ of assaults against nurses were associated with the adverse stimulation of patients, during the care process, and they recognized that patients' mental states might cause them to misinterpret non-aversive staff behaviour. However, they suggested that certain nurses behaved unprofessionally with the deliberate aim of adversely stimulating patients, e.g. by insulting, threatening or criticizing them. This study elaborated on the work of (Lanza 1988, Morrison 1989). All of the authors investigated the association between patient aggression and the nursing role.

Finnema et al. (1996) analyzed the association between rule enforcement and the levels of high expressed emotion amongst staff. They hypothesized that nurses may create ward environments dominated by rules in order to manage 
difficult situations, and designed an educational programme to help them use alternative interventions with psychotic patients. They evaluated the impact of the programme on levels of high expressed emotion, and the number of ward rules. The authors found that the nurses' levels of high expressed emotion did not change, but the number of ward rules decreased. However, confounding factors could have caused the change, and the researchers did not use a control group. The study augmented Whittington $\&$ Wykes' (1996) findings in that high expressed emotion may evoke negative reactions from patients in much the same way as aversive stimulation, but high expressed emotion differs in that nurses may unconsciously rather than deliberately display these attitudes during interactions with patients.

\section{7}

Psychiatric nurses frequently impose rules in the denial of patients' requests. Nijman et al. (1997) investigated the circumstances surrounding aggressive incidents within a closed acute psychiatric unit. The authors used the Staff Observation of aggression Scale (SOAS) to record the location and characteristics of the incidents. The findings showed that out of 164 violent incidents, $32 \%$ were precipitated because the nurses denied the patients' requests, but there was no way of knowing whether other elements of nurse-patient interaction triggered aggression. Nijman et al. recognised that communication problems between staff and patients might have been an important cause of aggression. This study expanded on previous studies that explored the relationship between the frustration of patient autonomy and aggression (Lanza 1988, Morrison 1989, Roper \& Anderson 1991).

One of the categories in a qualitative study by Letendre (1997) showed that users held negative perceptions of the ward regime. They felt oppressed by the degree of control over their activities, and identified a number of extra rules that were not applied in the wider society or in other hospital wards.

Crichton (1997) analyzed the moral judgements made by nurses when patients break the rules. He used a case vignette design whereby nurses rated the management of an incident, and the scale included one question designed to measure perceived moral responsibility. Nurses were asked whether they felt that rule breaking was driven by mental disorder, free choice or lack of self control. Crichton identified a censorious element in the responses, which raised questions about the regulation of nurses' reactions towards non-compliant patients. Crichton recommended the development of a system that acknowledged the influence of moral judgements, and the incorporation of procedures that might ensure justifiable responses to patient misdemeanour. He acknowledged that the vignette design isolated patients' behaviour from the context, but did not consider that nurses' personal experience of violence might influence the degree of censure. Crichton's recommendations reflected Richardson's (1995) ideas, but he focused on the role of nurses in making moral judgements, and Richardson focused on formalising responses after the decision was made. Both studies made an important point about the need to regulate institutional responses to patient misdemeanour because arbitrary or subjective responses may lead to the inappropriate or punitive imposition of ward rules and sanctions.

\section{8}

A comparative study by Crichton et al. (1998) compared the attitudes of Canadian and British nursing staff towards the management of patient misdemeanour. The nurses were shown case vignettes, and completed a semi-structured questionnaire. The Canadian results mirrored the findings in Crichton's (1997) study in that responses involving moral censure were perceived as more useful. The results showed that the use of medication and seclusion received higher ratings in the Canadian sample. Conversely, talking and relaxation techniques were rated higher in the British sample. Crichton et al. acknowledged that differences in the data collection methods, and in the work experience of the participants may account for these results, but the authors also hypothesized that differences in the base rate of serious violence in North America might be a factor.

Crichton (1998) discussed how psychodynamic theory might help the staff to understand and respond to rule breaking. Classical dynamic theory gives a perspective on internal control and rule keeping.

He draws a parallel between the encouragement of compliance with psychiatric ward rules and the parental function, and discusses how patients' previous experiences of poor parenting may be re-enacted by the institution if the staff are not helped to manage the feelings engendered by rule breaking. This paper elaborates on the work of Jurgensen (1971), Levinson \& Crabtree (1979), Watkins (1979), and Kologjera et al. (1989). Crichton's recommendations are similar, but like Johansen (1983) he advocates rule flexibility in certain circumstances.

The effect of a culture of control on the behaviour of nurses is pertinent to a study of ward rules. Morrison (1998) used causal modelling to test the hypothesis that staff might display rigid attitudes towards patients if they perceived that organisational structures neglected personal development and relationship issues in favour of control. She identified satisfaction with the hospital as the predom- 
inant emergent variable, and found that satisfied staff laid less emphasis on rules. She found that males were more authoritarian than females, and nurses from lower socioeconomic groups were more controlling than those from higher groups. She concluded that rigidity might stem from a combination of individual characteristics and organizational factors, but did not seem to consider that staff from lower socio-economic groups might not have high levels of qualifications or skills, and yet might be in most contact with patients, which could cause them to be more restrictive. Greater exposure to patient aggression amongst male nurses might result from social expectations of the male role, and this might account for the higher incidence of this variable in Morrison's analysis of the data. The study augmented the work of previous authors on the authoritarian attitudes of ward-based nurses (Alden 1978, Roper \& Anderson 1991, Hewison 1995).

\section{9}

There is evidence that patients abscond because of the restrictions placed upon them when they are in a hospital. In a prospective study of absconding, Bowers et al. (1999) interviewed patients who absconded from acute admission wards. This study linked absconding, which could be viewed as a form of rule breaking with the insensitive application of ward rules, and with the trivialization of patients' requests. The role of psychiatric nurses in evoking aggression and other negative reactions in patients during the performance of their duties emerged in several of the studies that have been reviewed (Roper \& Anderson 1991, Whittington \& Wykes 1996).

Crichton (1999) examined staff attitudes towards disruptive behaviour in group homes for people with learning disability. Moral judgement emerged as a major factor. Appearance degree of learning disability and apparent danger were major variables in staff con captions of the degree to which residents were judged to be morally responsible and culpable residents. This study augmented Crichton's (1997) findings in which he also recommended stronger management systems to counter the effect of censoriously driven responses to patient misdemeanour. Crichton acknowledges the limitations of the case vignette design, which divorced the incident from its social context, but he did not seem to consider that staff that had experienced violence might give more censorious responses.

\section{2}

Mistral et al. (2002) analyzed the impact of therapeutic community principles on a high-care, 14-bed ward for the management of detained patients. The interventions incor- porated improvements in communication regarding the aims and clarity of ward rules. Clear rules and sanctions were created, and were communicated to all staff and patients. The rules related to smoking, alcohol, the use of illegal substances, and communal responsibility for the maintenance of the environment. Staff morale improved and the intervention had a positive effect on the functioning of the ward. The main areas of improvement were in rule clarity, mutual respect between staff and patients, better communication and an improved physical environment. However, the authors acknowledged that these improvements might be difficult to sustain once the intervention phase ended. This study augmented previous research that advocated rule clarity (Morrison 1989, Bensley et al. 1995).

\section{3}

Lowe et al. (2003) used case scenarios of real conflict events, which were rated by nurses. The results indicated that limit setting and structure were perceived as vital by psychiatric nurses, but that they could not be isolated from interventions that demonstrated respect for patients' autonomy. Differences in judgements between nurses of different status emerged from the results, and higher grades of staff were significantly more likely to favour autonomy confirming interventions.

\section{Discussion}

Several themes emerged from the research. Many studies highlighted an association between nurse-patient interaction and ward rules. Arguments about the importance of clear communication, rule clarity and consistency emerged from the literature. The absence of these factors was linked to patient aggression. Most of the studies provided no rich textual information about the actual content of nursepatient interaction.

Several studies advocated high structure to modify aggression, but an almost equal number concluded that rigid environments engender patient violence. The literature is divided in respect of the pros and cons of high structure, and needs to be more firmly grounded in evidence. This division of opinion means that we cannot establish whether rigid or flexible environments are the best way of managing psychiatric wards. The psychodynamic articles provide an alternative perspective on the use of ward rules. The authors argued that highly structured environments provide a corrective emotional experience, particularly for patients with personality disorder. What appears to be missing is a study that identifies the specific rule that is being implemented together with the antecedents and consequences. 
The issue of sanctions was explored, but only one study considered whether rule imposition is ethically justifiable or not in terms of patient outcomes. The ethical conflicts experienced by nurses in carrying out prescribed care for patients was analyzed by one researcher, but it is surprising that there are no studies focusing on the ethics of rule implementation. This issue may be highly sensitive because a study of ward rules highlights the relationship between punishment and treatment within psychiatry.

The potential to abuse ward rules to elicit patient compliance is not fully explored, and this is where issues about treatment and punishment converge. The boundaries between the treatment of psychiatric disorder and the control of behaviour are often blurred. A study designed to explore the ethical decision-making processes of nurses in relation to rule imposition and rule breaking might illuminate this grey area. Only one study was found that explored the relationship between race and rule implementation. A study focusing on the association between ethnicity and ward rules might help to define a framework that might embrace the differing cultural backgrounds of patients.

The view that patients are not just objecting to rule imposition during violent incidents emerges from the literature, and so does the argument that patient aggression is not solely produced by psychopathology. Several researchers intimated that the way in which nurses' approach patients during rule imposition is important in the prevention of aggression. The studies indicated that patients might refuse to comply and become aggressive when the rules are imposed in an insensitive and punitive manner. However, patients may view rules positively, if they perceive that the motivation for rule implementation arises from concern for their welfare.

The data related to ward rules tended to be group diverse behaviours, and most failed to identify specific aspects of nurse-patient interaction. Therefore, information about the rule that was being implemented and interactive elements are lost. This review has identified a gap in the literature in relation to a specific and comprehensive study of ward rules. However, the evidence points to an important relationship between ward rules and patient violence, and to a link between nurse-patient interaction and rule implementation. The findings reviewed above do not lead to sufficiently firm conclusions upon which to base patient care. An in-depth study of ward rules could address the important issues raised in this review of the literature.

\section{References}

Alden L. (1978) Treatment environment and patient improvement. Journal of Nervous and Mental Disorders 166, 327-334.
Aubrey T., Bradley L., Siddique C. \& Leblanc A. (1996) Program development on an acute in-patient psychiatric unit. Journal of Mental Health 5, 507-514.

Bensley L., Nelson N., Kaufman J., Silverstein B. \& Shields J. (1995) Patient and staff views of factors influencing assaults on psychiatric hospital employees. Issues in Mental Health Nursing 16, 433-446.

Bowers L., Jarrett M., Clark N., Kiyimba F. \& McFarlane L. (1999) Absconding: why patients leave. Journal of Psychiatric and Mental Health Nursing 6, 199-205.

Bursten B. \& Geach B. (1976) Ward policy and therapeutic outcome: review of patients' records. Journal of Nervous and Mental Diseases 163, 414-419.

Bursten B., Fontana A., Dowds B. \& Geach B. (1980) Ward policy and therapeutic outcome ratings of patient behaviour. Hospital \& Community Psychiatry 31, 33-37.

Caplan C. (1993) Nursing staff and psychiatric patients perspective of the ward atmosphere in a maximum security forensic hospital. Archives of Psychiatric Nursing 7, 23-29.

Crichton J. (1997) The response of nursing staff to psychiatric inpatient misdemeanour. Journal of Forensic Psychiatry 8, 3661.

Crichton J. (1998) Staff response to psychiatric inpatient violence: an international comparison. Psychiatric Care 5, 50-56.

Crichton J. (1999) Psychodynamic perspectives on staff response to patient misdemeanour. Criminal Behaviour and Mental Health 8, 266-274.

De Laune S. (1991) Effective limit setting how to avoid being manipulated. Nursing Clinics of North America 26, 757765.

Department of Health (2001) The Mental Health Policy Implementation Guide. Department of Health, London.

Finnema E., Louwerens J., Slooff C. \& van-den-Bosch R. (1996) Expressed emotion on long-stay wards. Journal of Advanced Nursing 24, 473-478.

Flaherty J., Naidu J., Lawton R. \& Pathak D. (1981) Racial differences in perception of ward atmosphere. American Journal of Psychiatry 138, 815-857.

Hewison A. (1995) Nurses' power in interactions with patients. Journal of Advanced Nursing 21, 75-82.

Johansen K. (1983) The impact of patients with chronic character pathology on a hospital inpatient unit. Hospital and Community Psychiatry 34, 843-847.

Jungman L. \& Bucher R. (1967) Ward structure, therapeutic ideology, and patterns of patient interaction. Archives of General Psychiatry 17, 407-415.

Jurgensen K. (1971) Limit setting for hospitalised adolescent psychiatric patients. Perspectives in Psychiatric Care. IX, 173178.

Kologjera L., Bedi A., Watson W. \& Meyer A. (1989) Impact of therapeutic management on use of seclusion and restraint with disruptive adolescent inpatients. Hospital and Community Psychiatry 40, 280-285.

Lanza M. (1988) Factors relevant to patient assault. Issues in Mental Health Nursing 9, 239-257.

Lanza M., Kayne H., Hicks C. \& Milner J. (1994) Environmental characteristics related to patient assault. Issues in Mental Health Nursing 15, 319-335.

Letendre R. (1997) The everyday experience of psychiatric hospitalisation: the users' viewpoint. International Journal of Social Psychiatry 43, 285-297. 
Levinson D. \& Crabtree L. (1979) Ward tension and staff leadership in a therapeutic community for hospitalised adolescents. Psychiatry 42, 220-240.

Lowe T., Wellman N. \& Taylor R. (2003) Limit-setting and decision making in the management of aggression. Journal of Advanced Nursing 41, 154-161.

Lutzen K. (1990) Moral sensing and ideological conflict aspects of the therapeutic relationship in psychiatric nursing. Scandinavian Journal of Caring Science 4, 69-76.

Mistral W., Hall A. \& McKee P. (2002) Using therapeutic community principles to improve the functioning of a high care psychiatric ward in the UK. International Journal of Mental Health Nursing 11, 10-17.

Moos R. (1974) The Ward Atmosphere Scale Manual. Consulting Psychologists Press, Palo Alto, CA.

Morales E. (1995) Least restrictive measures: alternatives to fourpoint restraints and seclusion. Journal of Psychosocial Nursing $33,13-16$.

Morrison E. (1989) Theoretical modeling to predict violence in hospitalised psychiatric patients. Research in Nursing and Health 12, 31-40.

Morrison E. (1994) The evolution of a concept: aggression and violence in psychiatric settings. Archives of Psychiatric Nursing VIII, 245-253.

Morrison E. (1998) The culture of care giving and aggression in psychiatric settings. Archives of Psychiatric Nursing 12, 2131.
Muir-Cochrane E. \& Harrison B. (1996) Therapeutic interventions associated with the seclusion of acutely disturbed individuals. Journal of Psychiatric and Mental Health Nursing 3, 319-325.

Nijman H., Allertz W., Merckelbach H., a Campo J. \& Ravelli D. (1997) Aggressive behaviour on an acute psychiatric admissions ward. European Journal of Psychiatry 11, 106-114.

Olsen D. (1997) When the patient causes the problem: the effect of patient responsibility on the nurse-patient relationship. Journal of Advanced Nursing 26, 515-522.

Pilgrim D. \& Rogers A. (1994) Service users' views of psychiatric nurses. British Journal of Psychiatric Nursing 3, 16-18.

Richardson G. (1995) Openness, order and regulation in a therapeutic setting. In: Psychiatric Patient Violence Risk and Response (ed Crichton, J.), pp. 97-111. Anthony Rowe Ltd, Chippenham Wiltshire.

Roper J. \& Anderson N. (1991) The interactional dynamics of violence, part 1: an acute psychiatric ward. Archives of Psychiatric Nursing 4, 209-215.

Taylor D. \& Taylor J. (1989) Mental Health in the 1990s from Custody to Care. Office of Health Economics, London.

Thomas E. (1996) Rethinking acute inpatient care. Australian and New Zealand Journal of Mental Health Nursing 5, 32-39.

Watkins T. (1979) Staff conflicts over use of authority in residential setting. Child Welfare 58, 205-215.

Whittington R. \& Wykes T. (1996) Aversive stimulation by staff and violence by psychiatric patients. British Journal of Clinical Psychology 35, 11-20. 
Copyright of Journal of Psychiatric \& Mental Health Nursing is the property of Blackwell Publishing Limited and its content may not be copied or emailed to multiple sites or posted to a listserv without the copyright holder's express written permission. However, users may print, download, or email articles for individual use. 